\title{
Minimally Invasive Approach in Bilateral Chronic Subdural Hematomas: Surgical Selection and Outcome in 74 Cases
}

\author{
Min $\mathrm{XU}^{1 *}$, Bo $\mathrm{YU}^{2}$, Cunzu WANG ${ }^{2}$, Xun $\mathrm{ZHU}^{3}$, Xiaofeng $\mathrm{LU}^{3}$, Pin $\mathrm{CHEN}^{4}$, Hengzhu ZHANG ${ }^{2}$ \\ ${ }^{1}$ Kunshan Affiliated Hospital of Nanjing University of Chinese Medicine, Kunshan Hospital of Traditional Chinese Medicine, \\ Department of Neurosurgery, Kunshan Jiangsu Province, China \\ ${ }^{2}$ Northern Jiangsu People's Hospital, Department of Neurosurgery, Yangzhou Jiangsu, China \\ ${ }^{3}$ Jiangsu University, School of Clinical Medicine, Zhenjiang Jiangsu, China \\ ${ }^{4}$ Northern Jiangsu People's Hospital, Yangzhou Jiangsu, China \\ ${ }^{*} \mathrm{Min} \mathrm{XU}$ and Bo YU are contributed equally to this work.
}

\section{ABSTRACT}

AIM: To investigate the effect of minimally invasive approaches on the outcome of bilateral chronic subdural hematoma.

MATERIAL and METHODS: A retrospective analysis was performed in 74 hospitalized bilateral chronic subdural hematoma patients from January 2010 to January 2013 in Northern Jiangsu People's Hospital. Patients' gender, age, hematoma location, history of trauma, surgical approaches, the operation time, hospitalized time and follow-up three months after discharge were recorded. Prognostic indicators including symptom relief and post-hospital neuro-imaging findings were extracted. Statistical methods were conducted to evaluate surgical efficacy.

RESULTS: Both twist drill and burr-hole surgeries had a satisfying symptomatic relief (84.93\%). Twist drill needs less operation time $(p<0.001)$ and less hospitalized time $(p=0.001)$. Maximal width of hematoma in computed tomography $(C T)$ findings was significantly different between twist drill group and burr-hole group $(p=0.026)$. Maximal width in CT findings was significantly associated with follow-up outcomes $(p=0.021)$.

CONCLUSION: Twist drill is a safe and effective minimally invasive surgery, especially for elderly patients with bilateral chronic subdural hematoma and a reasonable surgical operation seems to have more significant impact upon the surgical outcome.

KEYWORDS: Bilateral, Chronic subdural hematoma, Twist drill craniotomy, Burr-hole craniotomy, Outcome

\section{INTRODUCTION}


hronic subdural hematoma $(\mathrm{CSDH})$ is one of the most common clinical diseases in neurosurgical work, and the overall rate was about 14.1 in 100,000 persons per year in general population $(3,12,18)$. In developing countries, the incidence reached $0.0074 \%$ in elderly group (5). As a large percent of $\mathrm{CSDH}$, bilateral lesions had been reported to be approximately $20 \%(6,16)$. Due to the rising of life expectancy and the introduction of auxiliary tools such as computed tomography (CT) and magnetic resonance imaging (MRI), the higher incidence and diagnostic innovation provide a new cogitation for our clinical practice.

At present, there are many treatment techniques, such as conservative treatment, twist drill craniotomy, minicraniectomy or burr-hole, craniotomy or craniectomy and so on $(1,2,17,21)$. Early intervention and minimally invasive are often recommended, just as our center does. These treatment protocols are conducted with methods not requiring general 
anaesthesia, especially suitable for elderly patients. Most clinicians consider that there is no difference between bilateral $\mathrm{CSDH}$ and unilateral CSDH in the manifestations or treatment strategies (8). Despite of the similarities, bilateral CSDH still has its own feature. Treatment protocols and surgical details are altered by clinical symptoms, neuro-imaging findings and disease courses in bilateral CSDH.

In this study, a retrospective investigation of 74 bilateral CSDHs was conducted to investigate the effect of minimally invasive approaches on the outcome of bilateral CSDH.

\section{MATERIAL and METHODS}

\section{Patient Collection}

In the present study, we collected 74 bilateral CSDHs from January 2011 to January 2014 in Northern Jiangsu People's Hospital. The inclusion standards: all the patients were bilateral CSDHs and minimally invasive approaches mainly including burr holes and twist drill craniotomy. Conservative treatments and conventional craniotomy were excluded. Patients' gender, age, hematoma location, history of trauma, imaging finding, surgical approach, the operation time, hospitalized time, CT scans three months after leaving hospital were recorded. Prognostic indicators including symptom relief and posthospital neuro-imaging findings were extracted to evaluate surgical efficacy. Our research has been approved by Northern Jiangsu People's Hospital Institutional Review Board (IRB), and all clinical investigations have been conducted according to the principles expressed in the Declaration of Helsinki.

\section{Bilateral CSDH Classification}

Density of CSDHs is variable, especially bilateral ones. All the CT and MRI scans were reviewed and assessed by the surgeons after his 5 years' work experience. Due to the complexity of MRI imaging, the bilateral CSDHs were classified into four groups on the basis of CT scans (14) in this study: bilateral hypodensity, bilateral homogeneous isodensity or relative homogeneous high-density or bilateral different homogeneous densities, layered type, and mixed type (Figure 1A-D).
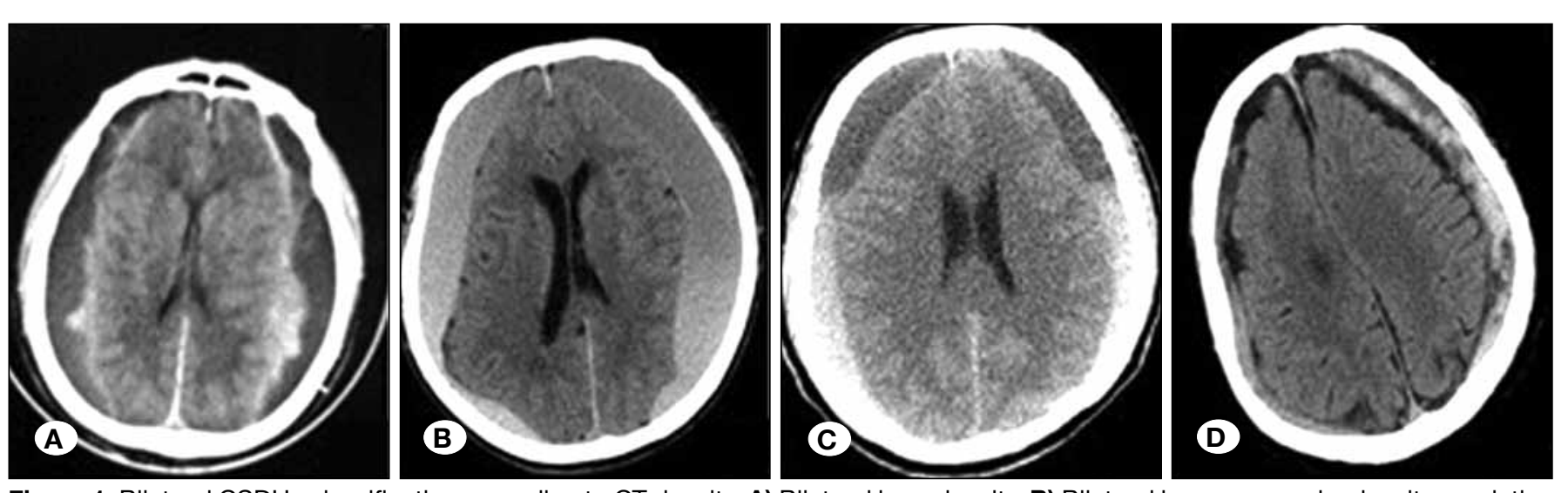

Figure 1: Bilateral CSDHs classification according to CT density. A) Bilateral hypodensity, B) Bilateral homogenous isodensity or relative homogenous high-density or bilateral different homogenous densities, C) Layered type, D) Mixed type.

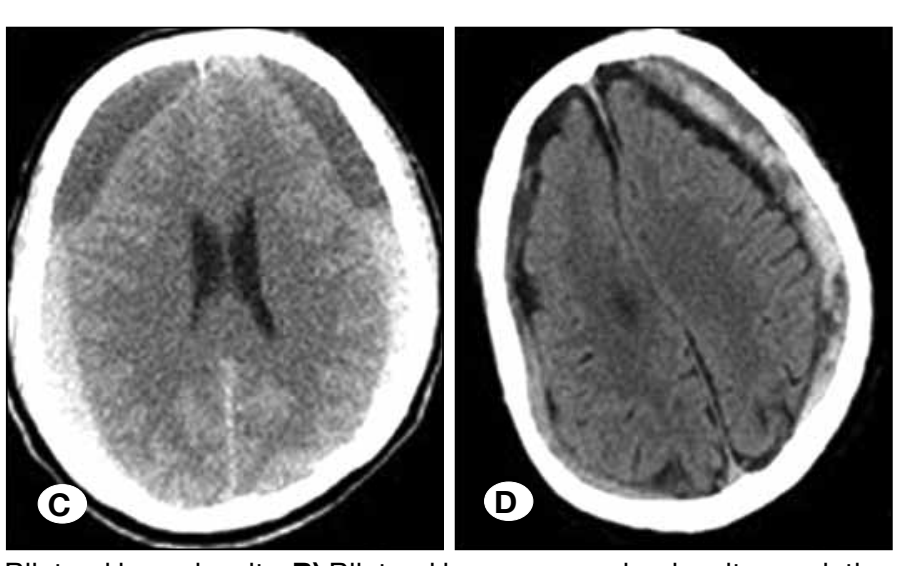

\section{Twist Drill Procedures}

Twist drill was selected as first-line treatment by most neurosurgeons, while a minority of surgeons relied on minicraniectomy or multiple burr-holes. The length of puncture needle is usually $20 \mathrm{~mm}$ (11 kinds of specifications, vary from 20-70 mm), which can be adjusted by rubber spacer. Briefly, on the basis of CT and MRI scans, a small incision was made under sterile conditions. A mini-craniectomy was made by using a hand drill on the maximal width of the cranial to prevent the catheter from entering the cortex. Then several rounds of flushing with sterile water helped to thin the hematoma and to facilitate drainage. The drainage was stopped when the hematoma was adequately drained or the drainage fluid became obviously clear. Follow-up CT scans were obtained the morning after the procedure to verify positioning and to determine the efficacy of the procedure.

\section{Statistical Analysis}

The variables were coded as qualitative or quantitative and analyzed by SPSS17.0 statistical package. A descriptive analysis was performed on the entire patient sample. Nonparametric rank sum test and $\chi^{2}$ test (with Yates correction when indicated) were used to compare categorical qualitative variables. Data was expressed as Median \pm Quartile and $p$-value of less than 0.05 was considered significant.

\section{RESULTS}

\section{Patients}

The eligible 74 patients were $69.5 \pm 18.25$ (13-86, Median is 69.5 ) years old; 8 patients (10.81\%) were older than 80 years old, 29 patients (39.20\%) between 70 to 80 years old, 17 patients (22.97\%) from 60 to 70 years old; 62 , male; 12 female; bilateral frontotemporal and parietal, 34; bilateral frontotemporal and parietal-occipital, 4; bilateral frontotemporal, 4. Forty-five patients $(60.81 \%)$ had undoubted causes of head trauma (Figure 2). The history of trauma was mainly from 4 to 13 weeks. Motor vehicle accidents or falling, slipping were mainly causes.

Headache, dizziness and vomiting were chief complaints (59, $79.73 \%)$. Limb weakness $(38,51.35 \%)$ and bad-response 
$(11,14.86 \%)$ were also common clinical manifestations. CT classification: Nine patients (12.16\%), typing A; twenty-seven patients (36.49\%), typing B; eleven patients (14.86\%), typing C; twenty-seven patients (36.49\%), typing D.

\section{Surgical Selection}

A 43-year-old man treated with conservative treatment was excluded. The remaining 73 patients were cured by minimally invasive approaches. They were classified as divided into two groups: patients in the method of twist drill (Twist drill group: $n=67$ ) and in burr-hole drainage (Burr-hole group: $n=6$ ). The follow-up three months after surgery was recorded and ranged from 1 to 3 points. Grade 1 represents symptomatic remission and the residual volume of hematoma is below $10 \%$, grade 2 represents symptomatic relief and the residual volume of hematoma is above $25 \%$, grade 3 represents existed clinical symptoms and the residual volume of hematoma is above $25 \%(19)$.

In the twist drill group, CT classification was distributed into typing $A$ ( 8 cases), typing $B$ (26 cases), typing $C$ (10 cases), and typing $D$ ( 23 cases). The maximal width of hematoma in CT findings was $20.64 \pm 6.88 \mathrm{~mm}$. The average operation time was $30.18 \pm 5.78$ minutes. Fifty-six patients $(83.58 \%)$ had a symptomatic relief. While in burr-hole group, CT classification was distributed into typing A (1 cases), typing B (1 cases), typing $C$ ( 1 cases), and typing $D$ ( 3 cases). The maximal width of hematoma in CT findings was $17.67 \pm 2.16 \mathrm{~mm}$. The average operation time and hospitalized time were $57.83 \pm 7.00$ minutes, $12.17 \pm 2.14$ days. All patients had a symptomatic relief.

Gender $(p=0.674)$, age $(p=0.912)$, history of head trauma $(p=0.147)$, CT classification $(p=0.532)$, and follow-up $(p=0.323)$ were shown non-significantly associated with these two minimally invasive approaches. However, there were significant group differences in CT findings $(p=0.026)$, the operation time $(p<0.001)$, and hospitalized time $(p=0.001)$ (Table I).

\section{Surgical Order in Twist Drill Surgery}

In the twist drill group, 35 patients accepted bilateral twist drill surgery simultaneously. On the basis of bilateral hematoma volume and surgical order in twist drill surgery, patients were divided into two groups: Less hematoma puncture group (the less side of hematoma volume was punctured first, then the more side), and more hematoma puncture group (the more

Table I: Group Difference of Clinical Parameters with Twist Drill Group and Burr-Hole Group

\begin{tabular}{|c|c|c|c|}
\hline & Twist drill (n=67) & Burr-hole $(n=6)$ & $\mathbf{p}$ \\
\hline Age (years) (Median \pm Quartile) & $69.00 \pm 18.00$ & $61.00 \pm 28.75$ & 0.912 \\
\hline History of head trauma (yes: no) & $43: 24$ & $2: 4$ & 0.147 \\
\hline CT classification (A: B: C: D) & $8: 26: 10: 23$ & $1: 1: 1: 3$ & 0.532 \\
\hline Hospitalized time & $6.42 \pm 1.37$ & $12.17 \pm 2.14$ & 0.001 \\
\hline Follow-up $(1: 2: 3)$ & $24: 32: 11$ & $3: 3: 0$ & 0.323 \\
\hline
\end{tabular}

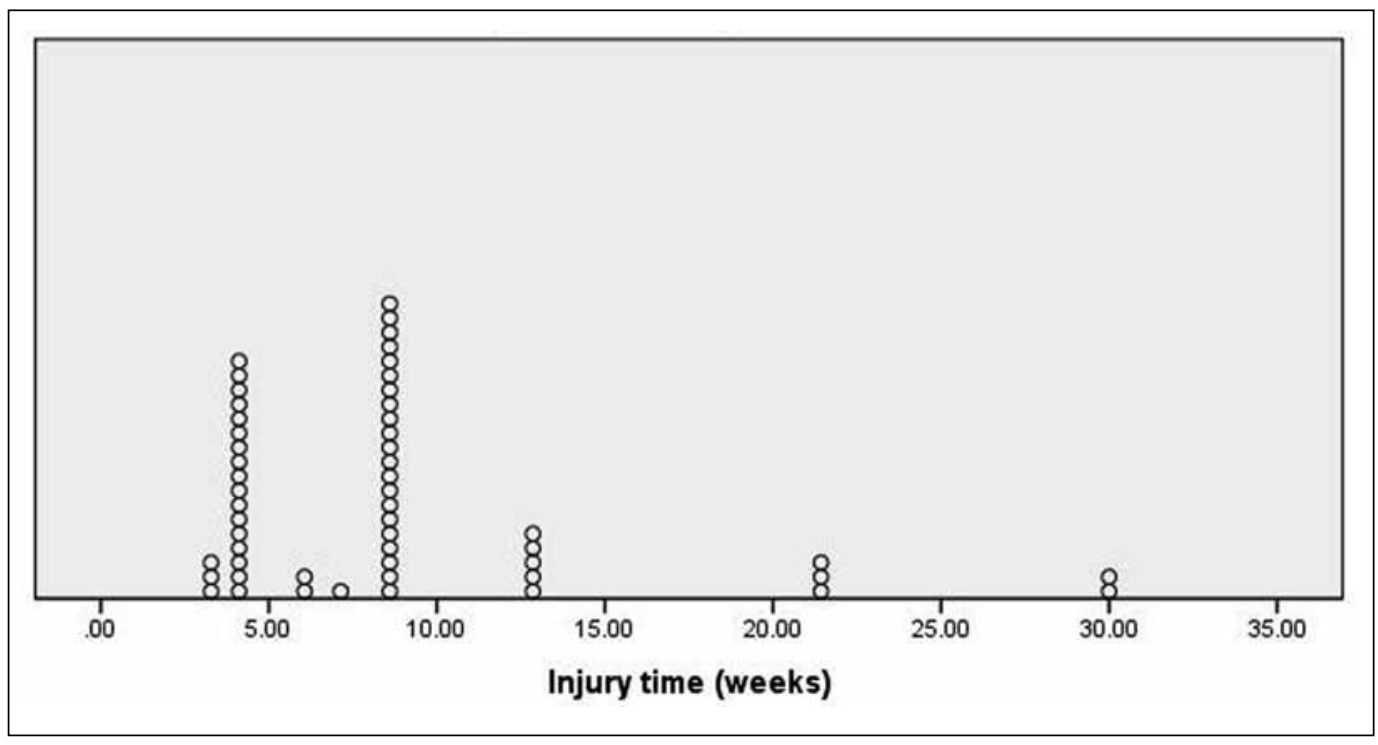

Figure 2: History of trauma. 
hematoma volume side was punctured first, then the less side). Thirty-three patients (94.29\%) had a definite mass effect on imaging findings: 13 with midline shift and pressed lateral ventricle, while 20 without obviously shift of midline structure. The mass effects of 30 patients were removed and 28 patients had a symptomatic relief three months after surgery.

Gender $(p=0.519)$, age $(p=0.564)$, history of head trauma $(p=0.394)$, CT classification $(p=0.578)$, maximal width in CT findings $(p=0.815)$, the operation time $(p=0.739)$, and hospitalized time $(p=0.337)$ were shown non-significantly associated with these two surgical orders. There was also non-significant group difference at three-month follow-up $(p=0.364)$ (Table II).

\section{Outcome After Minimally Invasive Surgery and Effect Factors}

Sixty-two patients $(84.93 \%$; 27 patients belonged to grade 1 , 35 patients belonged to grade 2) had a symptomatic relief. Eight patients had recurrence after surgery, and three patients had complications including epidural hematoma, fresh hematoma in hematoma cavity and brain parenchyma.

Gender $(p=0.558)$, age $(p=0.795)$, history of head trauma $(p=0.519)$, CT classification $(p=0.959)$, urokinase $(p=0.815)$, surgical approaches $(p=0.815)$, the operation time $(p=0.686)$, and hospitalized time $(p=0.931)$ were shown to be nonsignificantly associated with outcomes. Maximal width in CT findings was significantly associated with follow-up outcomes $(p=0.021)$ (Table III).

\section{DISCUSSION}

\section{Surgical Selection}

Based on clinical symptoms and neuro-imaging findings, patients after admission were entered into the hospitalized flowchart (Figure 3). Most patients had strong chief complaints or obvious mass effects on CT scans. Surgical treatments were taken into primary consideration. Among surgeries, burr holes and twist drill with drainage were two common minimally invasive methods curing CSDHs $(4,7,15)$, and recommended as efficient choices for uncomplicated CSDH (10). In our center, fifty-four patients were older than sixty years old. Compared with conservative treatments and craniostomy, measures above were preferred, and a relative satisfactory result (Sixty-two patients, $84.93 \%$ ) was obtained. Both types of surgical techniques seem effective for the treatment of $\mathrm{CSDH}(7,10)$. Type of surgical technique may not be a main variable improving the outcome of $\mathrm{CSDH}$ patients and may similarly act as a confounding factor (9) in bilateral CSDHs.

Table II: Group Difference of Clinical Parameters with Surgical Orders in Twist Drill Surgery

\begin{tabular}{lccc}
\hline & Group La $(\mathbf{n}=\mathbf{2 0})$ & Group $\mathbf{M}^{\mathrm{b}} \mathbf{( n = 1 5 )}$ & $\mathbf{p}$ \\
\hline Gender (male: female) & $17: 3$ & $12: 3$ & 0.519 \\
\hline Age (years) (Median \pm Quartile) & $68.50 \pm 17.00$ & $72.00 \pm 15.00$ & 0.564 \\
\hline History of head trauma (yes: no) & $14: 6$ & $12: 3$ & 0.394 \\
\hline CT classification (A: B: C: D) & $4: 9: 4: 3$ & $2: 7: 1: 5$ & 0.499 \\
\hline Maximal width in CT findings (mm) & $20.27 \pm 8.30$ & $20.87 \pm 6.00$ & 0.815 \\
The operation time & $32.95 \pm 4.32$ & $33.53 \pm 5.58$ & 0.739 \\
Hospitalized time & $6.55 \pm 1.10$ & $6.20 \pm 1.01$ & 0.337 \\
\hline Follow-up (1:2:3) & $8: 9: 3$ & $4: 7: 4$ & 0.364 \\
\hline
\end{tabular}

${ }^{a}$ Group L: Less hematoma puncture group; ' Group M: More hematoma puncture group.

Table III: Effect Factors of Minimally Invasive Surgery in Bilateral CSDH

\begin{tabular}{lcccc}
\hline & Grade 1 $\mathbf{( n = 2 7 )}$ & Grade 2 $(\mathbf{n}=\mathbf{3 5})$ & Grade 3 $(\mathbf{n}=\mathbf{1 1})$ & $\mathbf{p}$ \\
\hline Gender (male:female) & $22: 5$ & $29: 6$ & $10: 1$ & 0.558 \\
\hline Age (years) (Median \pm Quartile) & $72.00 \pm 20.00$ & $69.00 \pm 17.00$ & $67.00 \pm 18.00$ & 0.795 \\
\hline History of head trauma (yes: no) & $17: 10$ & $23: 12$ & $5: 6$ & 0.519 \\
\hline CT classification (A: B: C:D) & $3: 9: 7: 8$ & $4: 15: 3: 13$ & $2: 3: 1: 5$ & 0.959 \\
\hline Maximal width in CT findings (mm) & $20.15 \pm 5.75$ & $19.97 \pm 5.60$ & $26.09 \pm 10.05$ & $\mathbf{0 . 0 2 1}$ \\
The operation time & $32.67 \pm 9.42$ & $32.74 \pm 10.91$ & $31.00 \pm 5.29$ & 0.686 \\
Hospitalized time & $6.78 \pm 2.12$ & $7.03 \pm 2.20$ & $6.73 \pm 2.15$ & 0.931 \\
\hline Urokinase (yes: no) & $19: 8$ & $15: 20$ & $9: 2$ & 0.711 \\
\hline Surgery (1:2) & $24: 3$ & $32: 3$ & $11: 0$ & 0.364 \\
\hline
\end{tabular}


In the twist drill group, possible general anesthesia and tracheal intubation in burr-hole group were avoided. Local anesthesia was used, which shortened the total operating time and hospitalized time, and appeared to be beneficial to elderly patients (Table I). Fifty-six patients, $83.58 \%$, had a symptomatic relief.

\section{Surgical Order in Twist Drill Surgery}

The maximal width of hematoma in CT findings was $20.27 \pm 8.30 \mathrm{~mm}$, while the length of puncture needle was usually $20 \mathrm{~mm}$, which could be adjusted by rubber spacer. On the basis of clinical symptoms and neuro-imaging findings, thirty-five patients accepted bilateral twist drill surgery simultaneously. During the operation, surgical order is inevitable. Twenty-eight patients had a symptomatic relief and there was non-significantly group difference in three-month follow-up (Table II). When encountering definite mass effect or obvious midline shift on imaging findings, the more hematoma volume side ought to be punctured first. After drainage of hematoma, the reduction of intracranial pressure (ICP) makes it difficult to puncture on the other side of crania (20). Therefore, before successful puncturing in the less hematoma volume side, the drainage tube should be clipped without excessive drainage. Then continuous irrigation and closed drainage were conducted on both sides.

In contrast, when the mass effect or intracranial hypertension is not obvious, the less side of hematoma volume should be punctured first, then the more side. Similarly, excessive drainage may aggravate midline shift or bring hernia. Drainage tube needs to be clipped so as to avoid failure puncture or possible postoperative complication like epidural hematoma.

\section{Outcome After Minimally Invasive Surgery and Effect Factors}

The pathophysiology of bilateral CSDH is not well understood, but it is reported that traumatic injury leads to subdural hemorrhage. In our study, forty-five patients (60.81\%) had undoubted causes of head trauma. Several risk factors including age (more than 75 years old), coagulopathy, use of antiplatelet or anticoagulation medications, and hemodialysis were identified for bilateral CSDH (13). However, our analysis did not identify most risk factors mentioned above. Gender, age, history of head trauma, CT classification, and urokinase were all not significant risks (Table III).

Introduction of modern imaging methods and operative techniques improves the diagnosis and treatment of bilateral $\mathrm{CSDH}$ (11). Through CT and MRI, puncture point can be accurately located. The maximal width in CT findings or hematoma volume was only a factor affecting prognosis in our study. Further studies are required to assess the association between effect factors and recurrence.

In addition, this study has several limitations. The most important limitation is the retrospective study design and the reliance on medical records. Data collection and imaging reviews are less complete and accurate than a planned research. Most patients underwent twist drill surgery, while the sample in the burr-hole group was too small. The assessment of neurological status requires detailed classification.

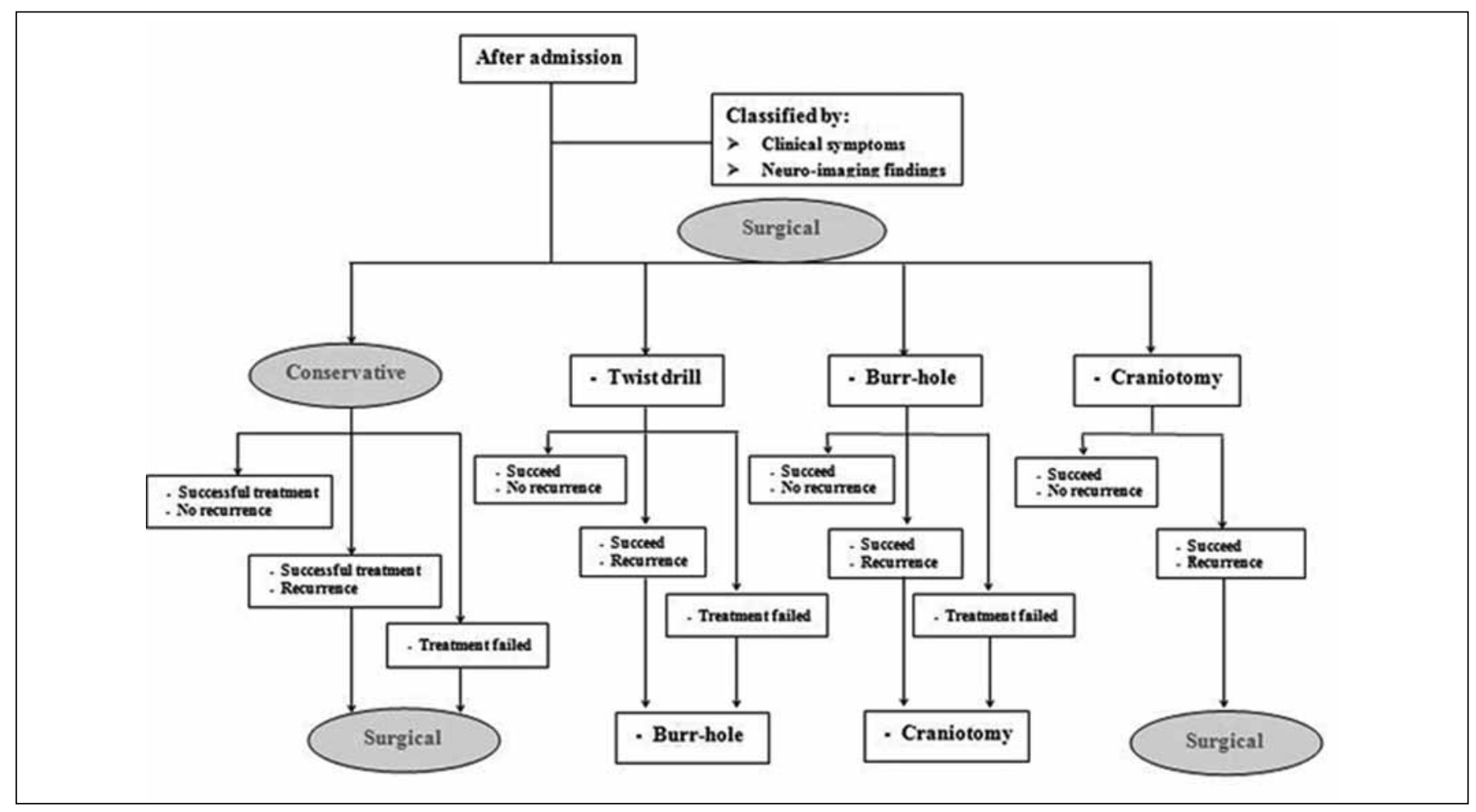

Figure 3: Hospital flowchart. 


\section{CONCLUSION}

Despite the limitations, this study provides useful information to identify surgical selection and measures in bilateral CSDH, which can evaluate prognosis and establish therapeutic strategies.

\section{REFERENCES}

1. Adhiyaman V, Asghar M, Ganeshram KN, Bhowmick BK: Chronic subdural haematoma in the elderly. Postgrad Med $\mathrm{J}$ 78(916):71-75, 2002

2. Algorta M, Spagnuolo E: Hematoma subdural crónico. Modalidades detratamiento: Revisión del tema. Propuestas de manejo. Rev Argent Neuroc 24:195-205, 2010

3. Aoki N: Chronic subdural hematoma in infancy. Clinical analysis of 30 cases in the CT era. J Neurosurg 73(2):201-205, 1990

4. Bozkurt G, Ayhan S, Akbay A, Palaoglu S: Treatment of chronic subdural hematoma by twist drill craniostomy with irrigation. Turk Neurosurg 16:19-24, 2006

5. Cousseau DH, Echevarria MG, Gaspari M, Gonorazky SE: Chronic and subacute subdural haematoma: An epidemiological study in a captive population. Rev Neurol 32(9):821-824, 2001

6. De Jesus $\mathrm{O}$, Pacheco $\mathrm{H}$, Negron B: Chronic and subacute subdural hematoma in the adult population. The Puerto Rico experience. P R Health Sci J 17(3):227-233, 1998

7. Gokmen M, Sucu HK, Ergin A, Gokmen A, Bezircio LH: Randomized comparative study of burr-hole craniostomy versus twist drill craniostomy; surgical management of unilateral hemispheric chronic subdural hematomas. Zentralbl Neurochir 69(3):129-133, 2008

8. Huang YH, Yang KY, Lee TC, Liao CC: Bilateral chronic subdural hematoma: What is the clinical significance? Int $\mathrm{J}$ Surg 11(7):544-548, 2013

9. Javadi A, Amirjamshidi A, Aran S, Hosseini SH: A randomized controlled trial comparing the outcome of burr-hole irrigation with and without drainage in the treatment of chronic subdural hematoma: A preliminary report. World Neurosurg 75(56):731-736, discussion 620-623, 2011
10. Lega BC, Danish SF, Malhotra NR, Sonnad SS, Stein SC: Choosing the best operation for chronic subdural hematoma: A decision analysis. J Neurosurg 113(3):615-621, 2010

11. Markwalder TM: Chronic subdural hematomas: A review. J Neurosurg 54(5):637-645, 1981

12. Miranda LB, Braxton E, Hobbs J, Quigley MR: Chronic subdural hematoma in the elderly: Not a benign disease. $J$ Neurosurg 114(1):72-76, 2011

13. Oyama H, Ikeda A, Inoue S, Shibuya M: The relationship between coagulation time and bilateral occurrence in chronic subdural hematoma. No To Shinkei 51(4):325-330, 1999

14. Park HR, Lee KS, Shim JJ, Yoon SM, Bae HG, Doh JW: Multiple densities of the chronic subdural hematoma in CT scans. J Korean Neurosurg Soc 54(1):38-41, 2013

15. Reinges $\mathrm{MH}$, Hasselberg I, Rohde V, Kuker W, Gilsbach JM: Prospective analysis of bedside percutaneous subdural tapping for the treatment of chronic subdural haematoma in adults. J Neurol Neurosurg Psychiatry 69(1):40-47, 2000

16. Robinson RG: Chronic subdural hematoma: Surgical management in 133 patients. J Neurosurg 61(2):263-268, 1984

17. Santarius T, Kirkpatrick PJ, Ganesan D, Chia HL, Jalloh I, Smielewski P, Richards HK, Marcus H, Parker RA, Price SJ, Kirollos RW, Pickard JD, Hutchinson PJ: Use of drains versus no drains after burr-hole evacuation of chronic subdural haematoma: A randomised controlled trial. Lancet 374(9695):1067-1073, 2009

18. Santarius T, Kirkpatrick PJ, Kolias AG, Hutchinson PJ: Working toward rational and evidence-based treatment of chronic subdural hematoma. Clin Neurosurg 57:112-122, 2010

19. Singla A, Jacobsen WP, Yusupov IR, Carter DA: Subdural evacuating port system (SEPS)--minimally invasive approach to the management of chronic/subacute subdural hematomas. Clin Neurol Neurosurg 115(4):425-431, 2013

20. Suzuki K, Sugita K, Akai T, Takahata T, Sonobe M, Takahashi S: Treatment of chronic subdural hematoma by closed-system drainage without irrigation. Surg Neurol 50(3):231-234, 1998

21. White M, Mathieson CS, Campbell E, Lindsay KW, Murray L: Treatment of chronic subdural haematomas - a retrospective comparison of minicraniectomy versus burrhole drainage. $\mathrm{Br}$ J Neurosurg 24(3):257-260, 2010 